\title{
Less toxic method for producing giant paper organ sections for pathology and anatomy education
}

This article was published in the following Dove Press journal:

Pathology and Laboratory Medicine International

17 November 2014

Number of times this article has been viewed

\author{
Nicole C DeMartinis \\ Walter E Finkbeiner \\ Department of Pathology, University \\ of California, San Francisco, \\ San Francisco, CA, USA
}

Purpose: Traditionally, medical school pathology educators have used formalin-fixed specimens to demonstrate effects of diseases on target organs, despite the handling of these "wet" tissues having distinct disadvantages, such as the need for gloves, protective clothing, and appropriate facilities to limit potential fixative moisture and fumes. Paper-mounted sections of solid organs have significant potential as an aid for teaching gross pathology and eliminate the disadvantages of handling formalin-fixed specimens. However, published techniques for preparing giant organ sections include the use of the highly toxic ethylene glycol monoethyl ether (EGEE; 2-ethoxyethanol). We investigated whether replacing EGEE with a less toxic dehydrating and clearing agent, Histo-Clear ${ }^{\mathrm{TM}}$, would allow production of high-quality sections.

Materials and methods: Whimster's procedure for preparing rapid paper sections of lungs and other organs was modified to incorporate the xylene substitute Histo-Clear in place of EGEE.

Results: Giant paper sections of various organs were prepared. In addition to lungs, excellent pathology teaching specimens were prepared from other organs, including the brain, heart, kidney, liver, and colon. Side-by-side comparisons of paper-mounted organ sections prepared using EGEE and Histo-Clear were indistinguishable. The sections prepared using Histo-Clear showed fine anatomical and pathological details.

Conclusion: The Gough-Wentworth technique of preparing rapid giant paper sections of organs was made less toxic without sacrificing quality by using Histo-Clear as an alternative to EGEE. Paper-mounted sections offer a safe and portable way for studying macroscopic pathology, and have great potential for use in the anatomy and pathology classroom, as well as in postgraduate pathology training.

Keywords: organ macrosections, macroscopic pathology, Gough-Wentworth sections

\section{Introduction}

In 1949, Gough and Wentworth described a technique to prepare giant papermounted sections of lungs to better observe and assess lung emphysema. ${ }^{1}$ Subsequently, Whimster modified the technique, shortening preparation time and extending its use to other solid organs. ${ }^{2}$ Further modifications have been suggested, but all currently described methods of preparing paper-mounted organ sections still include the use of the highly toxic ethylene glycol monoethyl ether (EGEE; 2-ethoxyethanol). ${ }^{3}$ We investigated whether replacing EGEE with a less toxic dehydrating and clearing agent, Histo-Clear ${ }^{\mathrm{TM}}$ (National Diagnostics, Atlanta, GA, USA), would allow production of high-quality sections. Substituting Histo-Clear for EGEE produced paper-mounted sections equivalent to Whimster's modification of
Correspondence: Walter E Finkbeiner Department of Pathology, San Francisco General Hospital - Building 3,

Room 205B, I00I Potrero Avenue, San Francisco, CA 94II0, USA

Tel +l 4I5 2068230

$\mathrm{Fax}+\mathrm{I} 4152065988$

Email walter.finkbeiner@ucsf.edu
Pathology and Laboratory Medicine International 20I4:6 4I-45 
the original Gough-Wentworth technique. We suggest that paper-mounted sections of solid organs have significant potential as an aid for teaching gross pathology.

\section{Materials and methods}

\section{Fixation}

Solid organ specimens were fixed with $10 \%$ formalin for at least 48 hours, though a few specimens had been preserved in formalin for over 20 years. In some cases, specimens were immersed in $100 \%$ ethyl alcohol to partly restore color.

\section{Embedding}

The embedding-solution formula was modified from Whimster's substituting Histo-Clear for EGEE (Table 1). The gelatin was slowly added to heated water in a beaker on a stirring plate. The best results were obtained when the gelatin and water solution was made within 2 days of embedding and then stored at $40^{\circ} \mathrm{C}-50^{\circ} \mathrm{C}$. Keeping the solution longer produced inferior freezing and slicing results. The Histo-Clear, capryl alcohol, and thiomersal were mixed into solution just prior to embedding. The specimen was transferred from formalin to water prior to embedding. While the specimen was soaking, a $1 \mathrm{~cm}$ layer of the embedding solution was poured into the embedding container. We used metal baking tins and freezer-safe plastic containers. Specimens were embedded in containers that were slightly larger than the specimens, thus saving reagents, limiting excess gelatin, and facilitating slicing. Next, the container was placed in the freezer for a short amount of time and removed when the layer felt tacky and not yet completely frozen. The specimens were removed from the water, and any excess fluid was gently squeezed out. The specimen was placed as flat as possible onto the tacky embedding-solution layer in the container. More embedding solution was poured into the container, but not enough to completely cover the specimen. The purpose of this step and having a base layer of almost frozen embedding solution was to prevent the specimen from floating and causing uneven orientation while freezing. Typically, the side of

Table I Embedding-solution formula: amounts usually sufficient for embedding three lung specimens

\begin{tabular}{ll}
\hline Chemical & Amount \\
\hline Histo-Clear & $165 \mathrm{~mL}$ \\
Capryl alcohol & $20 \mathrm{~mL}$ \\
I\% thiomersal & $14 \mathrm{~mL}$ \\
Gelatin (75-100 bloom) & $680 \mathrm{~g}$ \\
Water & $4,000 \mathrm{~mL}$ \\
\hline
\end{tabular}

Table 2 Mounting-solution formula: amount of solution usually mounted for 15-20 sections

\begin{tabular}{ll}
\hline Chemical & Amount \\
\hline Histo-Clear & $30 \mathrm{~mL}$ \\
Glycerin & $70 \mathrm{~mL}$ \\
I\% thiomersal & $10 \mathrm{~mL}$ \\
Gelatin (75-100 bloom) & $75 \mathrm{~g}$ \\
Water & $1,000 \mathrm{~mL}$ \\
\hline
\end{tabular}

the specimen placed down upon the prefrozen embedding solution produced better slices. The container was returned to the freezer, and when the gelatin mix became tacky once again, a final layer of embedding solution was added to completely cover the specimen. The organ blocks were allowed to freeze at $-20^{\circ} \mathrm{C}$ overnight before slicing. Keeping the blocks frozen for longer periods did not affect results.

\section{Slicing}

The frozen blocks were removed from the freezer and allowed to thaw slightly to facilitate slicing. However, with excessive thawing, the blocks became less rigid and produced incomplete slices. A block that becomes overly thawed can be simply placed back in the freezer before slicing. Slices (2-3 mm) were prepared using a professional meat slicer (1612P; Hobart, Troy, OH, USA). Once cut, the slices were carefully placed into $10 \%$ formalin, aiding removal of excess gelatin. After 5 minutes, the slices were transferred to water. After being floated in water for 10-15 minutes, the slices were placed in fresh $10 \%$ formalin just prior to mounting. If mounting was postponed, the organ slices were stored in formalin.

\section{Mounting}

The modified mounting solution (Table 2) was prepared and used immediately and never stored. Again, Histo-Clear

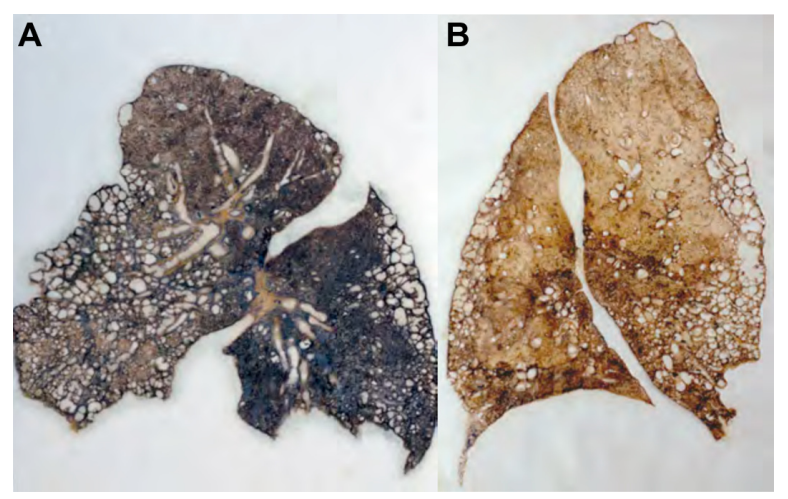

Figure I (A) Idiopathic pulmonary fibrosis specimen prepared using ethylene glycol monoethyl ether. (B) Idiopathic pulmonary fibrosis specimen prepared using Histo-Clear. 

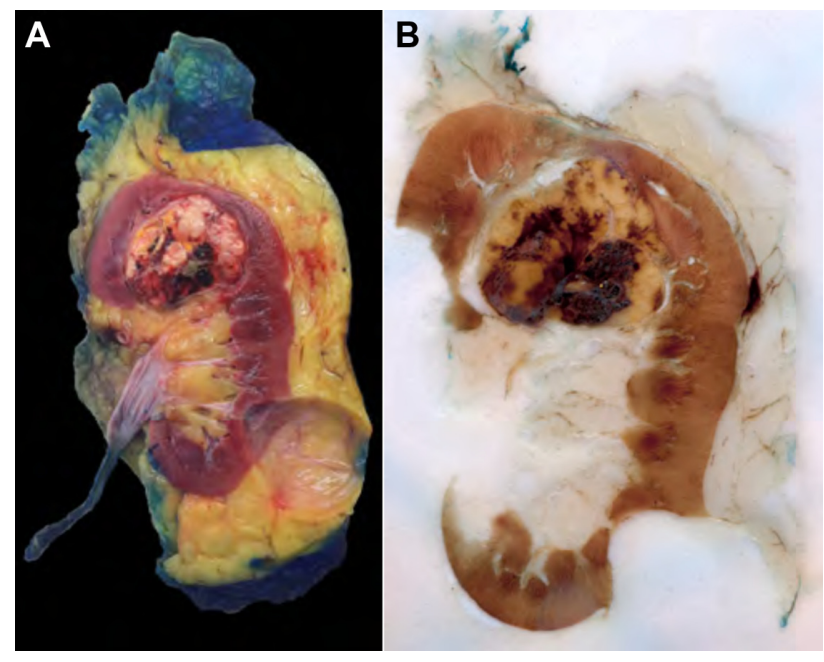

Figure 2 (A) Surgical resection of an unfixed kidney specimen showing a renal cell carcinoma and simple cortical cyst. (B) Paper-mounted section of same specimen prepared following formalin fixation. The blue color on the specimen is ink used to mark the margin of resection.

was substituted for EGEE. A rigid, acrylic plastic sheet was lightly covered with the mounting solution. A sliced section was removed from the formalin bath and placed on the sheet. However, for brain specimens, which tended to stick to the plastic, a sheet of BioDesignGelWrap ${ }^{\mathrm{TM}}$ (BioDesign, Carmel, NY, USA) was placed onto the plastic prior to the specimen, and another layer of mounting solution was added on top of the BioDesignGelWrap. Typically, other organs did not require the BioDesignGelWrap. Next, the excess gelatin was carefully cut away from the specimen, and bubbles from the solution were gently pressed out. A thin layer of mounting solution was poured over the slice, and the plastic sheet was covered with $3 \mathrm{~mm}$ Whatman ${ }^{\circledR}$ chromatography paper (GE Healthcare BioSciences, Pittsburgh, PA, USA). The paper was lightly coated with the mounting solution as well, and then any new bubbles were again carefully pressed out, taking care not to tear the paper. Binder clips were placed onto the edges of the wet paper mounted on the acrylic plastic sheets to prevent curling.

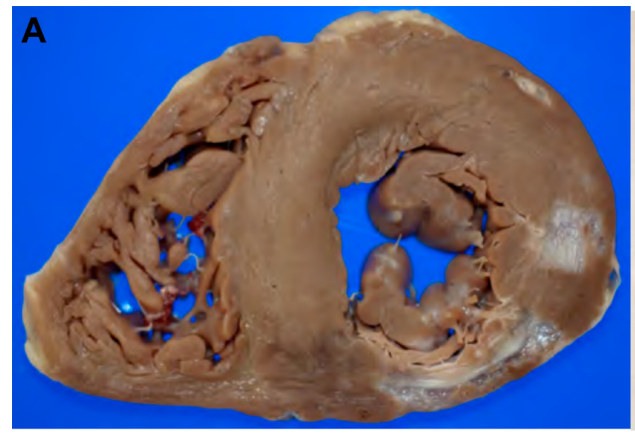

\section{Drying}

The paper-mounted organ sections were dried at room temperature. Once dry, the paper was easily peeled off the acrylic plastic sheet. To prevent curling of the organ sections, they were laminated in plastic as soon as possible. This also served to protect the sections without altering their appearance. For laminating, we used $10 \mathrm{~mm}$ laminating pouches and a heavy-duty thermal laminator (TLC 7020 Pouch Laminator; Oregon Laminations, Portland, OR, USA).

\section{Results}

Side-by-side comparisons of paper-mounted organ sections prepared using EGEE and Histo-Clear were indistinguishable. Both methods revealed fine anatomical and pathological details. Figure 1 shows two lung specimens with idiopathic pulmonary fibrosis prepared separately with EGEE and Histo-Clear. Both demonstrate pulmonary fibrosis and architectural remodeling that has led to prominent "honeycomb" cystic changes. Figure 2 compares an unfixed kidney specimen showing a renal cell carcinoma and cortical cyst with the paper-mounted section of the same specimen. There are obvious differences in color between the unfixed and fixed specimen; however, both show the fine details of the neoplasm and the normal cortical and medullary renal parenchyma to excellent advantage. Figure 3 compares a formalin-fixed section of heart with a remote myocardial infarct with the paper-mounted section of the same specimen.

The Gough-Wentworth technique has most notably been used for studying lungs with emphysema; however, the technique is useful for demonstrating the gross pathology of other lung diseases (Figure 4). In addition to lungs, teaching specimens were prepared from other solid organs, including brain, colon, heart, kidney, liver, spleen, and placenta. Representative samples are shown in Figure 5.

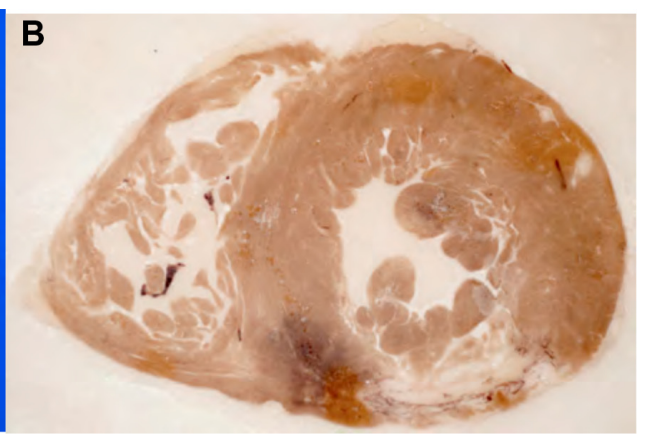

Figure 3 (A) Formalin-fixed section of heart with a remote myocardial infarct in the posterior wall of the left ventricle. (B) Paper-mounted section of same specimen. 

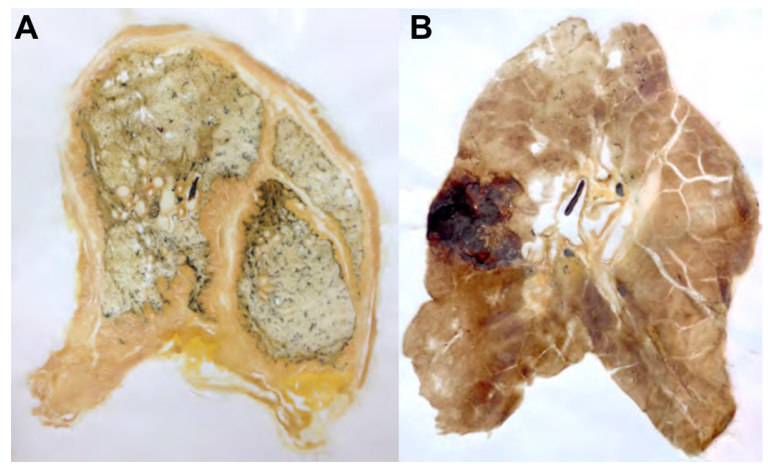

Figure 4 ( $A$ and B) Though originally devised for demonstrating pulmonary emphysema, the macroscopic features of other lung diseases are advantageously displayed in papermounted sections. (A) Paper-mounted section of a right lung with mesothelioma that had been stored for over 15 years. The lung parenchyma is gray, but the neoplasm is nicely demonstrated. (B) Paper-mounted section of a lung with a pulmonary infarct.

\section{Discussion}

The original method of preparing rapid giant paper sections was described in 1949 by Gough and Wentworth, and was intended for whole-lung sections. ${ }^{1}$ They used the sections to macroscopically examine lung disease, especially emphysema. The disadvantage of the original method was that the final sections were not ready for almost 2 weeks, and included so many steps that only a small number of sections could be produced at a time. Whimster introduced modifications that reduced the gelatin content and shortened the production of paper-mounted sections to 24-48 hours, thereby allowing production of greater numbers of sections more easily. ${ }^{2}$ Whimster also used the sections to study primarily the lung, but also extended their use to other solid organs, including the brain, thyroid, heart, liver, spleen, adrenal, kidney, and pancreas. By substituting the safer Histo-Clear for EGEE, we obtained comparable sections to the Whimster modification of the Gough-Wentworth method, while eliminating the most toxic reagent. Histo-Clear could not be directly substituted for the EGEE in the mounting solution, as that amount $(40 \mathrm{~mL})$ degraded the mounting paper. Therefore, the final suggested amount of Histo-Clear was less than EGEE. However, at this final concentration, the appearance of the organ sections was comparable, and the mounting paper remained intact. Histo-Clear II was also tested. When substituted directly for Histo-Clear, the sections prepared with this agent required longer drying times and consistently degraded the mounting paper. Reducing the concentration of the Histo-Clear II eliminated the paper degradation, but the drying times did not improve. Histo-Clear II is advertised as having a reduced citrus odor compared to Histo-Clear; however, we found that Histo-Clear II did not offer any significant advantages over Histo-Clear.
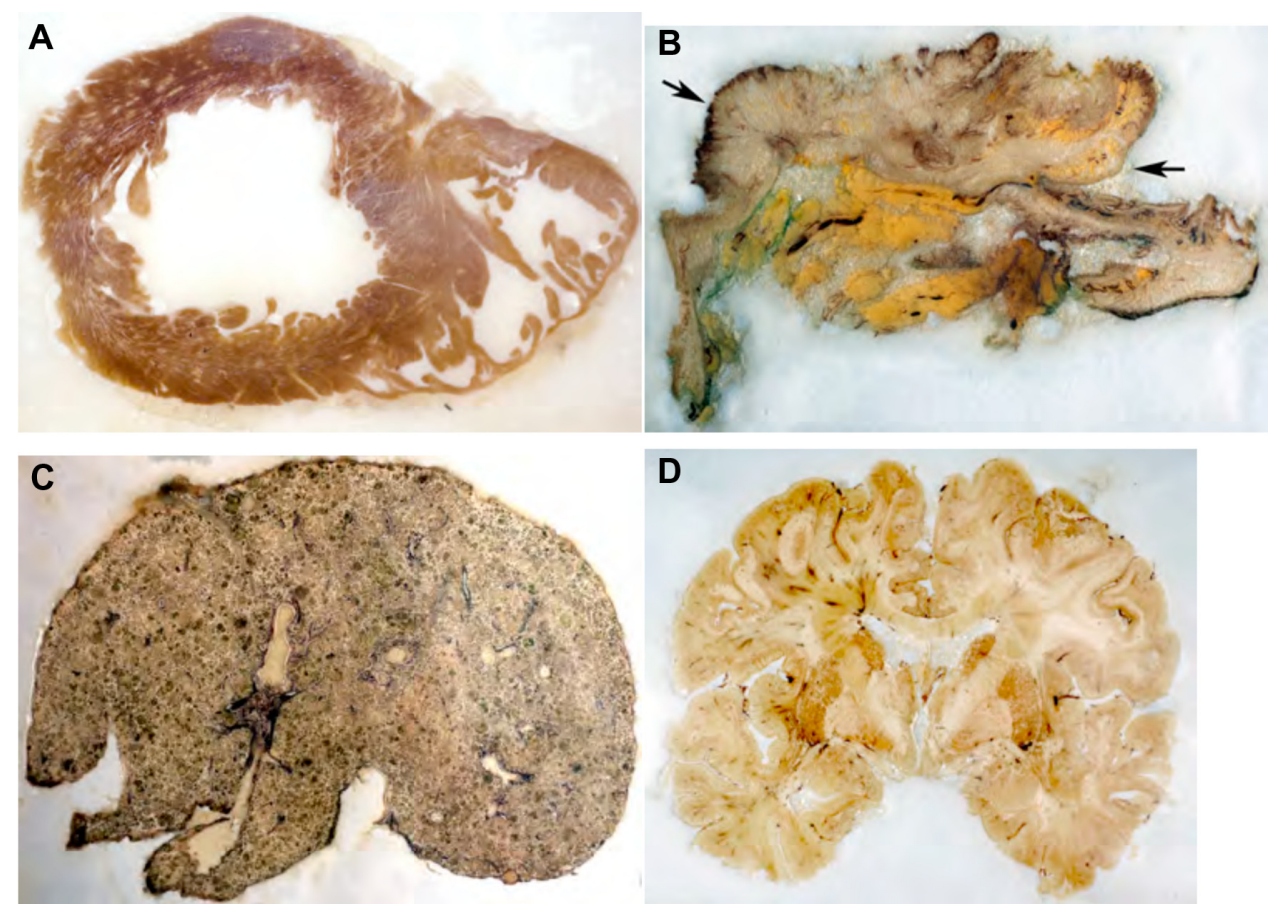

Figure 5 (A-D) Other examples of macroscopic pathology and anatomy demonstrated in paper-mounted organ sections. (A) Horizontal section of a heart through the right and left ventricle with eccentric hypertrophy due to rheumatic heart disease. (B) Surgical resection of a colonic adenocarcinoma. Though the starting tissue was small and did not yield numerous sections, the luminal neoplasm (arrows) is apparent. (C) Liver from an individual with alcoholic cirrhosis who died following hemorrhagic shock. The specimen shows acute hepatic necrosis and prominent fibrous scarring. (D) Paper-mounted coronal section of a normal brain made at the approximate level of the mammillary bodies. Permanent paper-mounted brain sections have obvious utility for teaching neuroanatomy. 
Other modifications of the Gough-Wentworth technique suggested that slices could be kept in formalin indefinitely after cutting if mounting cannot occur immediately. We found that delaying mounting sometimes altered the color of the specimens, though this varied with different organs. Immediate mounting produced the best result for most organs. However, keeping slices of brain in a formalin bath overnight made mounting these specimens easier, by reducing their fragility and aiding removal of excess gelatin.

To avoid having the specimens stick to the acrylic plastic sheets after being peeled, we tried a variety of techniques, including wax paper, cellophane, and BioDesignGelWrap. Ultimately, the BioDesignGelWrap produced the best results, though some degree of curling of the paper was inevitable. Curling was overcome by applying binder clips on the edges as drying. The best results were obtained when the sections were allowed to dry overnight at room temperature. We attempted to shorten the drying time by using an artificial heat source (hair dryer), but this ran the risk of overheating and shrinking the specimens.

Some paper sections would curl over time, despite being flat when initially peeled off the acrylic plastic sheet. Lamination should occur as soon as possible after removing the dry-paper section from the acrylic plastic sheet to avoid curling and best preserve the section. The final product should be a sturdy laminated paper section. We preferred $10 \mathrm{~mm}$ laminator film pouches, but thinner film can also be used.

\section{Conclusion}

The Gough-Wentworth technique of preparing rapid giant paper sections of organs was made less toxic without sacrificing quality by substituting Histo-Clear for EGEE. In addition to lungs, demonstration specimens were prepared from other solid organs, including the brain, heart, kidney, and liver. Giant paper-mounted organ sections have great potential for use in the anatomy and pathology classroom, as well as in postgraduate pathology training. These sections show fine anatomical and pathological details, and offer a safe and portable way for studying macroscopic pathology. Also, the finished, laminated product preserves the specimen for many decades, and can replace formalin-fixed, wet specimens or Plexiglas-encased museum pieces.

\section{Acknowledgment}

This project was supported in part by a 2014 UCSF Interprofessional Grant from the Joint Library/IPE Instructional Grants Program.

\section{Disclosure}

The authors report no conflicts of interest in this work.

\section{References}

1. Gough J, Wentworth JE. The use of thin sections of entire organs in morbid anatomical studies. J R Microsc Soc. 1949;69:231-235.

2. Whimster WF. Rapid giant paper sections of lungs. Thorax. 1969;24: 737-741.

3. Gevenois PA, Koob MC, Jacobovitz D, De Vuyst P, Yernault JC, Struyven J. Whole lung sections for computed tomographic-pathologic correlations. Modified Gough-Wentworth technique. Invest Radiol. 1993;28:242-246.
Pathology and Laboratory Medicine International

\section{Publish your work in this journal}

Pathology and Laboratory Medicine International is a peer-reviewed open access journal focusing on innovative basic research and translational research related to pathology or human disease. The journal includes original research, updates, case reports, reviews and commentaries on current controversies. The Academic Sponsor

\section{Dovepress}

of this journal is the Chinese American Pathology Association (CAPA). The manuscript management system is completely online and includes a very quick and fair peer-review system. Visit http://www.dovepress.com/testimonials.php to read real quotes from published authors. 